\title{
Readaptation to the laboratory in long-term sleep studies
}

\author{
E. STEPANSKI, T. ROEHRS, P. SAAB, F. ZORICK, and T. ROTH \\ Sleep Disorders and Research Center, Henry Ford Hospital, Detroit, Michigan 48202
}

\begin{abstract}
Readaptation to the sleep laboratory was assessed in 10 volunteers complaining of insomnia with polysomnographic evidence of disturbed sleep. After an initial 3-night adaptation to the laboratory, subjects slept in the laboratory on 2 consecutive nights per week for 10 weeks and were administered medication according to the following protocol: placebo, Week 1; triazolam (.5 mg), Weeks 2-7; and placebo, Weeks 8-10. Routine sleep recordings were obtained on all laboratory nights. Over the 10 weeks, there were no statistically significant differences (p > .05) between Nights 1 and 2 on the following sleep parameters: percentage wake time, percentage REM, latency to Stage 2, and number of awakenings. It was concluded that readaptation effects in long-term studies are relatively small. The minimal sleep disruption associated with readaptation does not outweigh the advantages of taking repeated laboratory samples of 1 or 2 nights.
\end{abstract}

It is well established that sleep on the initial night in the laboratory is not a reliable index of sleep on subsequent nights (Agnew, Webb, \& Williams, 1966; Dement, Kahn, \& Roffwarg, 1965; Rechtschaffen \& Verdone, 1964). As a result, laboratory studies of sleep usually include an adaptation period of 1 or 2 nights before data are collected. Long-term studies of sleep often use alternating series of nights in the laboratory and at home. It is not clear whether, after an initial adaptation to the laboratory, a readaptation night is necessary on each reentry to the laboratory after a number of nights at home.

The only two studies that have been done on laboratory readaptation have produced conflicting results. One study (Scharf, Kales, \& Bixler, 1975) used subjects with insomnia and reported that both entry to the laboratory and a single reentry after 7 nights at home had similar disruptive effects on sleep. Longer sleep latencies, lower percentages of REM and Stage 4 sleep, and increased total wake time were found. The other report (Thornby, Mauk, \& Karacan, 1979) studied normal subjects who reentered the laboratory on four occasions at 2-week intervals. While sleep was disrupted on the initial night in the laboratory, reentry to the laboratory had a minimal effect on sleep. Sleep efficiency, the ratio of time asleep to time in bed, and the number of awakenings did not change; only percentage REM was slightly suppressed.

The different results of these reports may be due to the different populations studied or the different methodologies used. In contrast to Thornby et al. (1979), Scharf et al. (1975) studied only a single night of readaptation, compared adaptation with placebo to readaptation with drug, and combined the adaptation and readaptation nights in data analyses. Since questions remain about the reliability of sampling 1 or 2 nights in the laboratory, rather than monitoring every night, it is necessary to systematically assess possible readaptation effects over an extended period. The present study used subjects with insomnia and assessed readaptation on 10 series of laboratory nights under both placebo and drug conditions.

\section{METHOD}

\section{Subjects}

Ten volunteers with a complaint of insomnia participated in the study. They ranged in age from 18 to 50 years (mean age = 29.9 years \pm 9.6 ). Each of the subjects was classified as DIMSpersistent psychophysiological (Association of Sleep Disorder Centers, 1979). The degree of sleep disturbance was objectively determined with 3 nights of prestudy polysomnography, which also served as the laboratory adaptation period. The criterion for inclusion into the study was defined as a sleep latency of longer than $30 \mathrm{~min}$ or a minimum of $60 \mathrm{~min}$ of wakefulness for 2 of the 3 screening nights.

\section{Procedure}

After the initial adaptation to the laboratory for 3 consecutive nights, subjects slept in the laboratory 2 consecutive nights per week for 10 weeks. They returned to the laboratory on the same nights of the week each time. Alcohol, drugs, and naps were prohibited during screening and the study.

The protocol was as follows: placebo, Week 1; triazolam $(.5 \mathrm{mg})$, Weeks 2-7; and placebo, Weeks 8-10. Medication was administered each night, $30 \mathrm{~min}$ before lights out. Subjects determined their own sleep times, which were held constant throughout the study. On laboratory nights, routine sleep recordings (Rechtschaffen \& Kales, 1968) were obtained.

\section{RESULTS}

The sleep parameters studied were those previously shown to be affected by laboratory adaptation and readaptation: percentage wake time, latency to Stage 2, number of awakenings, and percentage REM. A twofactor repeated-measures analysis of variance was per- 
Table 1

Means and Standard Deviations of Sleep Parameters on Night 1 and Night 2

\begin{tabular}{|c|c|c|c|c|c|c|c|c|c|c|c|c|c|c|c|c|}
\hline \multirow[b]{3}{*}{ Week } & \multicolumn{4}{|c|}{ Percent REM } & \multicolumn{4}{|c|}{ Percent Wake Time } & \multicolumn{4}{|c|}{ Latency to Stage 2} & \multicolumn{4}{|c|}{ Awakenings } \\
\hline & \multicolumn{2}{|c|}{ Night 1} & \multicolumn{2}{|c|}{ Night 2} & \multicolumn{2}{|c|}{ Night 1} & \multicolumn{2}{|c|}{ Night 2} & \multicolumn{2}{|c|}{ Night 1} & \multicolumn{2}{|c|}{ Night 2} & \multicolumn{2}{|c|}{ Night 1} & \multicolumn{2}{|c|}{ Night 2} \\
\hline & Mean & SD & Mean & $\mathrm{SD}$ & Mean & $\mathrm{SD}$ & Mean & SD & Mean & SD & Mean & $\mathrm{SD}$ & Mean & SD & Mean & $\mathrm{SD}$ \\
\hline 1 & 11.3 & 4.08 & 15.6 & 4.25 & 26.7 & 19.36 & 8.3 & 4.50 & 61.7 & 100.49 & 23.1 & 14.38 & 8.2 & 6.03 & 7.9 & 5.51 \\
\hline 2 & 11.2 & 4.78 & 15.0 & 4.00 & 13.2 & 11.91 & 6.9 & 6.59 & 17.1 & 16.19 & 12.6 & 7.41 & 8.6 & 5.08 & 5.8 & 3.71 \\
\hline 5 & 15.8 & 4.89 & 14.0 & 8.39 & 9.4 & 8.75 & 18.6 & 13.79 & 23.8 & 23.48 & 25.4 & 24.20 & 4.6 & 3.34 & 6.0 & 5.83 \\
\hline 6 & 14.8 & 5.63 & 16.1 & 7.16 & 10.3 & 9.65 & 10.6 & 13.91 & 16.1 & 11.66 & 19.4 & 11.16 & 6.3 & 6.68 & 7.5 & 13.19 \\
\hline 9 & 17.6 & 6.48 & 17.6 & 5.27 & 15.1 & 12.60 & 13.7 & 16.92 & 26.1 & 14.96 & 30.2 & 28.64 & 7.2 & 6.48 & 5.1 & 3.75 \\
\hline 10 & 14.1 & 7.80 & 14.2 & 5.45 & 16.9 & 18.60 & 18.3 & 16.29 & 23.0 & 19.87 & 39.8 & 38.22 & 5.9 & 3.81 & 6.3 & 4.22 \\
\hline Mean* & 14.6 & 6.06 & 15.3 & 5.77 & 14.9 & 16.49 & 11.3 & 11.54 & 31.3 & 57.38 & 23.5 & 21.05 & 6.6 & 5.20 & 6.5 & 6.25 \\
\hline
\end{tabular}

*Mean over all 10 weeks.

formed on each of these parameters. The first factor was Night 1 vs. Night 2, and the second factor was week of the study (10 weeks). There was no significant difference between Night 1 and Night 2, and the Night by Week interaction was not significant $(p>.05)$. The main effect due to weeks was significant for percentage wake time $[F(9,81)=4.96, p<.001]$ and latency to Stage $2[F(9,81)=2.34, p<.05]$. These changes over weeks can be attributed to administration of the hypnotic drug, triazolam, which was given 6 of the 10 weeks of the study.

Table 1 presents means and standard deviations of the sleep parameters on Nights 1 and 2 for selected weeks. During Week 1 , subjects received placebo, during Week 2, triazolam, during Weeks 5 and 6, chronic triazolam, and during Weeks 9 and 10, placebo. While there were no significant differences between Nights 1 and 2 overall, there was a slight disturbance of sleep on Night 1 of the first two series (Weeks 1 and 2) of laboratory nights. Latency to Stage 2 sleep and percentage wake time were increased, and percentage REM was decreased on Night 1 compared with that on Night 2. Thereafter, sleep of Nights 1 and 2 was similar on all parameters, regardless of drug condition.

\section{DISCUSSION}

The results of the present study show that readaptation to the sleep laboratory is not a significant factor in long-term studies particularly after one or two reentries to the laboratory. These results support the findings of Thornby et al. (1979), in normal subjects, showing that after several discrete series of nights in the laboratory, sleep on the 1st night of a series is not markedly disturbed. Further, the present results suggest that the difference between the results of Thornby et al. (1979) and those of Scharf et al. (1975) is due to difference in methodology. Thornby et al. assessed readaptation after several reentries to the laboratory, whereas Scharf et al. studied the first reentry to the laboratory. In the present study, sleep on the 1st night of the first reentry to the laboratory was slightly disturbed, compared with 1 st nights on later series.

The present results suggest that number of reentries to the laboratory is a factor to be considered in the design of longterm sleep studies. It is possible that some readaptation does occur on the first or second reentry to the laboratory. If evaluation of initial changes is an objective of a long-term study, that study might be designed with readaptation nights on the first or second series of laboratory nights. On the other hand, in studies in which initial changes are not in question, the added expense of readaptation nights does not seem warranted. In other words, after one or two reentries to the laboratory, a relatively reliable sample of sleep can be obtained on the 1st night of a series of laboratory nights.

Sleep researchers generally assume that after adaptation to the laboratory, sleep measures for subsequent nights in the laboratory are consistent (reliable) from night to night. Usually, no significant differences between means of various sleep parameters across nights are found. When consistency of sleep measures was evaluated using correlation coefficients between pairs of nights over 15 consecutive nights, mean coefficients ranged from .10 to .57 , depending on the sleep parameter (Roth, Kramer, \& Roehrs, 1976). Mean reliability coefficients between Night 1 and Night 2 for the present study were: percentage $\mathrm{REM}=.59$, percentage wake time $=.42$, latency to Stage $2=$ .26 , and number of awakenings $=.55$. These values are similar to those of Roth et al. (1976) and did not vary systematically over the 10 weeks. Although one must be cautious in making comparisons between studies, this comparison suggests that after adaptation, a sample of 2 nights will yield data with reliability similar to that found for any pair of nights taken from an extended series of laboratory nights.

A remaining question is what number of nights away from the laboratory will produce sleep disturbance on reentry to the laboratory. Thornby et al. (1979) found a minimal sleep disruption in normal subjects with approximately 14 days between the series of laboratory nights. In the present study, using subjects with insomnia, there were minimal readaptation effects with 5 days between series of laboratory nights. A complete study of laboratory readaptation will require the systematic variation of the interval of nights at home between series of laboratory nights. It should also be noted that the critical interval length may well vary depending on the population studied.

\section{REFERENCES}

Agnew, H. W., Webb, W. B., \& Williams, R. I. The first night effect: An EEG study of sleep. Psychophysiology, 1966, 2, 263-266.

Association of Sleep Disorder Centers. Diagnostic classification of sleep and arousal disorders (1st ed.). Sleep, 1979, 2, 1-137.

Dement, W. C., Kahn, E., \& Roffwarg, H. P. The influence of the laboratory situation on the dreams of the experimental subject. Journal of Nervous and Mental Disease, 1965, 140, 119-131.

Rechtschaffen, A., \& Kales, A. A manual of standardized terminology, techniques, and scoring systems for sleep stages of 
human subjects (Public Service Publication 204). Washington, D.C: U.S. Government Printing Office, 1968.

Rechtschaffen, A., \& Verdone, P. Amount of dreaming: Effects of incentive, adaptation to laboratory and individual differences. Perceptual and Motor Skills, 1964, 19, 947-958.

Roth, T., Kramer, M., \& Roenrs, T. The consistency of sleep measures. Sleep, 1976, 5, 286-288.

Scharf, M. B., Kales, A., \& Bixler, E. O. Readaptation to the sleep laboratory in insomniac subjects. Psychophysiology, $1975,12,412-415$.

Thornby, J. I., Mauk, J. L., \& Karacan, I. A further examination of the adjustment/readjustment-night phenomena. Sleep Research, 1979, 8, 152.

(Received for publication April 27, 1981.) 